\title{
physiospektrum
}

\section{Dokumentationszeit reicht hinten und vorne nicht}
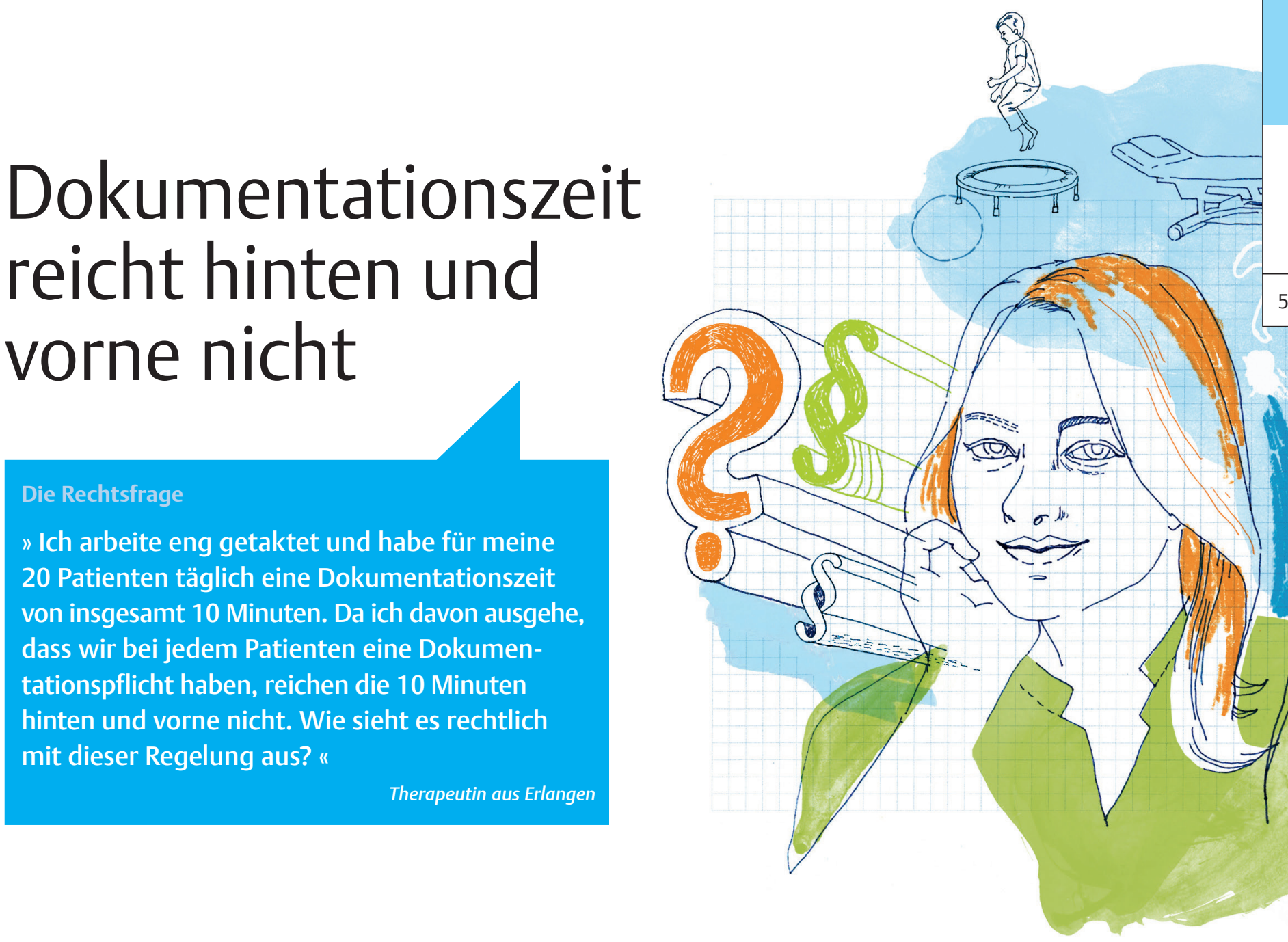

\section{Expertenantwort}

Grundsätzlich ist ein Therapeut laut Bürgerlichem Gesetzbuch zur Dokumentation verpflichtet. Dafür muss er sämtliche aus fachlicher Sicht wesentlichen Maßnahmen und deren Ergebnisse notieren. Diese gesetzliche Verpflichtung steht im Zusammenhang mit dem Behandlungsvertrag, der zwischen dem Praxisinhaber und dem Patienten geschlossen wird. Damit hat der Praxisinhaber gegenüber dem Patienten die Pflicht, die Behandlung ordnungsgemäß zu dokumentieren. Der Therapeut ist als Angestellter des Praxisinhabers aus rechtlicher Sicht ein sogenannter Verrichtungs- bzw. Erfüllungsgehilfe. Im Rahmen seines Arbeitsverhältnisses ist er gegenüber seinem Arbeitgeber bei jedem Patienten zu einer ordnungsgemäßen Dokumentation verpflichtet.

Eine Dokumentationszeit von insgesamt 10 Minuten für 20 Patienten am Tag kann nicht ausreichen - zumal vor der ersten Behandlung eine umfangreiche Befunderhebung erfolgen und ein therapeutischer Behandlungsplan erstellt werden muss.
Weist der Arbeitgeber den angestellten Therapeuten an, insgesamt nur 10 Minuten auf die Dokumentationen zu verwenden, ist dies arbeitsrechtlich zunächst möglich. Immerhin bestimmt der Arbeitgeber im Rahmen seines Direktionsrechtes, was der Arbeitnehmer wann, wo und wie lange tun soll. Zivilrechtlich haftet der Praxisinhaber gegenüber dem Patienten für die ordnungsgemäße Dokumentation aufgrund des mit ihm geschlossenen Vertrags. Der Arbeitnehmer hat die Nebenpflicht aus dem Arbeitsvertrag, seinen Arbeitgeber vor absehbaren Schäden zu bewahren. Hieraus entsteht in Ihrem Fall die Pflicht, den Arbeitgeber darauf hinzuweisen, dass die 10 Minuten für eine ordnungsgemäße Dokumentation aller Patienten eines Tages nicht ausreichen. Bleibt Ihr Chef trotzdem bei seiner Anweisung und weigert sich ausdrücklich, die zusätzlich für Dokumentationen verwendete Zeit als Arbeitszeit zu bezahlen, bleibt Ihnen nur übrig, weisungsgemäß circa neuneinhalb Minuten für eine ordnungsgemäße
Dokumentation zu verwenden. In den letzten etwa 30 Sekunden der Arbeitszeit erstellen Sie eine Liste, welche Behandlungen Sie nicht dokumentieren konnten, und händigen diese Aufstellung Ihrem Arbeitgeber aus. Denn: Unterlassen Sie es, auf die nicht dokumentierten Behandlungen hinzuweisen, riskieren Sie eine Abmahnung bzw. Kündigung. Das belastet sicher Ihr Arbeitsverhältnis, der Grund dafür ist jedoch alleine die zu enge Zeitvorgabe. Karsten Bossow

\section{$\rightarrow$ Wirft auch Ihr Berufsalltag rechtliche Fragen auf? Dann schreiben Sie an Simone.Gritsch@thieme.de.}

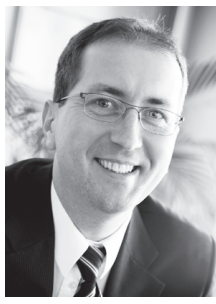

Karsten Bossow ist seit 1999 Rechtsanwalt. Seit 2003 ist er Fachanwalt für Arbeitsrecht und seit 2010 für Medizinrecht. 